\title{
Possible sarcopenia: early screening and intervention-narrative review
}

\author{
Wen-Qing Xie ${ }^{1,2 \#}$, Ge-Lei Xiao ${ }^{3 \#}$, Pei-Wu Hu${ }^{4}$, Yu-Qiong $\mathrm{He}^{5}$, Shan $\mathrm{Lv}^{6}$, Wen-Feng Xiao ${ }^{1,2}$ \\ ${ }^{1}$ Deparment of Orthopedics, Xiangya Hospital, Central South University, Changsha, China; ${ }^{2}$ National Clinical Research Center for Geriatric \\ Disorders, Xiangya Hospital, Central South University, Changsha, China; ${ }^{3}$ Department of Neurosurgery, Xiangya Hospital, Central South \\ University, Changsha, China; ${ }^{4}$ Department of Scientific Research, Xiangya Hospital, Central South University, Changsha, China; ${ }^{5}$ Deparment of \\ Surgery, Xiangya Hospital, Central South University, Changsha, China; ${ }^{6}$ Department of Geriatric Endocrinology, The First Affiliated Hospital of \\ Nanjing Medical University, Nanjing, China \\ Contributions: (I) Conception and design: WQ Xie, GL Xiao; (II) Administrative support: S Lv, WF Xiao; (III) Provision of study materials or \\ patients: All authors; (IV) Collection and assembly of data: PW Hu; (V) Data analysis and interpretation: YQ He; (VI) Manuscript writing: All \\ authors; (VII) Final approval of manuscript: All authors. \\ \#These authors contributed equally to this work. \\ Correspondence to: Dr. Wen-Feng Xiao. Department of Orthopedics, Xiangya Hospital, Central South University, Changsha, China. Email: \\ wenfeng_xiao@163.com; Dr. Shan Lv. Department of Geriatric Endocrinology, The First Affiliated Hospital of Nanjing Medical University, \\ Nanjing, China. Email: lvshan3366@126.com.
}

\begin{abstract}
Sarcopenia is a new geriatric syndrome that has become a heavily researched topic, and it is a potential risk factor for weakness, disability, and death in elderly people. As the world's population ages, the incidence of sarcopenia has also increased, which has resulted in a series of health problems and in large medical costs. Although there are generally accepted diagnostic criteria for sarcopenia, the existing criteria require a comprehensive evaluation of muscle quality, muscle strength and muscle function. Most of these evaluations are time-consuming, labourious, difficult to implement, and unsuitable for large-scale population surveys. Moreover, the abilities of the elderly to undertake daily-life activities are often affected when they are diagnosed with sarcopenia. Therefore, if individuals who are likely to suffer from sarcopenia could be identified by screening at an early stage and then comprehensively evaluated, time and labour would be saved, and the detection rate would be improved. Timely intervention can be undertaken in possible sarcopenia to prevent further development of sarcopenia and strongly improve the quality of life of individuals. This study reviews the early screening and intervention of the possible sarcopenia, analyses its advantages and disadvantages and attempt to identify reliable and practical methods to reduce adverse consequences and the extent of harm.
\end{abstract}

Keywords: Early intervention; early screening; sarcopenia

Submitted Apr 16, 2020. Accepted for publication Sep 16, 2020.

doi: 10.21037/apm-20-967

View this article at: http://dx.doi.org/10.21037/apm-20-967

\section{Introduction}

The word "sarcopenia" comes from the Greek words "sarco", meaning muscle (Greek sarx), and "penia", meaning loss; thus, "sarcopenia" means muscle loss. In 1989, Rosenberg (1) first proposed the word sarcopenia to describe a decrease in skeletal muscle mass with age.
Before 2010, the diagnosis of sarcopenia was mainly based on Baumgartner's relative skeletal muscle index (SMI) (2). However, this diagnostic standard neglects the evaluation of muscle strength and muscle function and considers only changes in muscle quality. In 2010, the European Working Group on Sarcopenia in Older People (EWGSOP) recommended that muscle mass, muscle strength, and 
muscle function should be simultaneously evaluated when diagnosing sarcopenia (3). Sarcopenia has subsequently been redefined as a disease that is mainly characterized by the decline of muscle mass, muscle strength, and muscle function. Comprehensive diagnostic criteria were also adopted for the diagnosis of sarcopenia (4). In September 2016, the World Health Organization (WHO) incorporated sarcopenia into ICD-10, which shows that sarcopenia was acknowledged internationally and has attracted widespread concern in society (5). Furthermore, the incidence of sarcopenia increases with age (6). In Asia, and depending on the use of diagnostic criteria proposed by different sarcopenia working groups, such as the EWGSOP, the International Working Group on Sarcopenia (IWGS), the Asian Working Group for Sarcopenia (AWGS), and the Foundation for the National Institutes of Health Sarcopenia Project (FNIH), the reported prevalence of sarcopenia fluctuates from $0 \%$ to $16.1 \%$ (7-9). In Europe, if the definition that provides the lowest prevalence estimate is used, the prevalence rate of sarcopenia is predicted to rise from $11.1 \%$ in 2016 to $12.9 \%$ in 2045 (10). In international studies, data up to 2014 show that $5-10 \%$ of people over 65 years old have sarcopenia (11). In addition, using the latest WHO age standard, $40-50 \%$ of the elderly ( $\geq 80$ years old) suffer from sarcopenia (12).

Sarcopenia can be caused by a variety of factors, including advanced age, neuromuscular degeneration, changes in muscle protein turnover, changes in hormone levels and sensitivity, chronic inflammation, oxidative stress and behavioral/lifestyle factors, endocrine diseases, and malignant tumours (13). The pathophysiology of sarcopenia is multifactorial, with reduced caloric intake, muscle fibre innervation, intracellular oxidative stress, hormonal decline, and increased myostatin signalling all being considered to be causes of this condition (14). The onset of sarcopenia is not only affected by risk factors at the time of onset but also by genetic and lifestyle factors of the whole life process. With ageing of the world's population becoming more severe, the incidence of sarcopenia will significantly increase and result in additional serious adverse consequences and harm.

There are many evaluation methods for the diagnosis of sarcopenia, such as dual energy X-ray absorptiometry (DXA), bioelectrical impedance assessment (BIA), computed tomography (CT), magnetic resonance imaging (MRI), and ultrasound (US), which are all employed for evaluating muscle quality. Testing hand grip strength, knee flexion and extension, and maximum expiratory flow rate are methods used to evaluate muscle strength (15). To evaluate muscle function, routine pace assessment, simple body function assessment, and the stand-up test, stand-up walking test, and long-distance walking test are regularly used $(15,16)$. However, the diagnosis of sarcopenia must be comprehensively based on three aspects: muscle quality, muscle strength, and muscle function. It is necessary to screen out individuals who may suffer from sarcopenia at an early stage, as most of these methods are time-consuming and labourious and need to be performed by professionally trained personnel. Early screening of sarcopenia is not only helpful in identifying individuals who may suffer from sarcopenia at an early stage but can also provide references for the development and prognosis of other diseases. Studies have shown that newly developed sarcopenia is also an independent negative predictor of survival after liver transplantation (17). Moreover, the use of age, grip strength, and calf circumference to screen sarcopenia can be used to predict future adverse events in patients with heart failure (18).

We present the following article in accordance with the NARRATIVE REVIEW reporting checklist (available at http://dx.doi.org/10.21037/apm-20-967).

\section{Early screening of sarcopenia}

Presently, there is no unified standard for the early screening of sarcopenia. The commonly used early screening methods in epidemiological investigation and clinical practice include SARC-F combined with calf Circumference (SARC-CalF), gait speed, body mass index (BMI) and US.

\section{SARC-F combined with calf circumference (SARC-CalF)}

As a relatively simple and inexpensive screening method, questionnaires are widely used in epidemiological investigations, especially in community screening. To overcome the difficulties of comprehensive sarcopenia diagnosis in a large-scale epidemiological investigation, Malmstrom and Morley (19) developed the SARC-F screening test. In SARC-F (Strength, Assistance with walking, Rise from a chair, Climb stairs, and Falls) questionnaire, each of the five items has 2 points for a total of 10 points, and a total score $\geq 4$ points indicates that the risk of sarcopenia is increased. The latest research on SARC-F shows that compared with diagnostic criteria proposed by authoritative sarcopenia-working groups, such as EWGSOP, IWGS, and FNIH, SARC-F has a higher 
detection overlap and higher specificity for the muscle function test, which is an excellent test for identifying muscle function damage and muscle atrophy $(20,21)$. Thus, SARC-F is a better screening test for functional measures and it is reasonable to regard SARC-F as the first simple step in the hierarchical screening process. Many studies have confirmed that SARC-F is a suitable tool for community screening of sarcopenia and is suitable for scientific research, experimental observations, and largescale community epidemiological investigations (22-25). The International Clinical Practice Guidelines for Sarcopenia (ICFSR) and EWGSOP2 also recommend SARC-F as the preferred method for the rapid screening of sarcopenia, even if SARC-F is less sensitive $(26,27)$. In clinical practice, EWGSOP2 advises the use of the SARC-F questionnaire to determine if individuals have probable sarcopenia. The sarcopenia defined by SARC-F can greatly improve the predictive value of clinical characteristics to predict adverse physical manifestations (28). Moreover, SARC-F is also a simple tool that can be implemented for postmenopausal women and individuals who need longterm care $(29,30)$. A stepwise model was used to assess the incremental predictive ability, and concluded that the fiveitem SARC-F questionnaire may be shortened to three items (strength, climb stairs, and assistance in walking) in the rapid screening for sarcopenia in clinical practice (SARC-F-3) (31). However, after comparing the specificity and sensitivity of SARC-F-3 and SARC-F, Yang et al. (32) concluded that SARC-F-3 might not be suitable for screening sarcopenia in the elderly. Therefore, it is still controversial whether SARC-F-3 can replace SARC-F as a simpler and more convenient early screening method for sarcopenia, and a large amount of medical evidence is needed for confirmation.

To improve the low sensitivity of the pure SARC-F test, Barbosa-Silva et al. proposed a new test method, SARCCalF, and claimed that SARC-CalF significantly improved the screening performance and sensitivity of SARC-F for sarcopenia and could be used in clinical practice (33). Moreover, calf circumference (CC) may also be a good indicator for screening sarcopenia among the elderly in South Korea, and the optimal cut-off values of CC for low muscle mass are $35 \mathrm{~cm}$ for men and $33 \mathrm{~cm}$ for women (34). In addition, the AWGS 2019 update proposes separate algorithms for community $v s$. hospital settings, which both begin by screening either calf circumference $(<34 \mathrm{~cm}$ in men and $<33 \mathrm{~cm}$ in women), SARC-F $(\geq 4)$, or SARC-CalF $(\geq 11)$ to facilitate earlier identification of people at risk for sarcopenia (35). SARC-CalF is the best choice to screen sarcopenia according to a comparison with SARC-F and other screening tools such as the Mini Sarcopenia Risk Assessment in both the full version (MSRA-7) and the short version (MSRA-5) (36-38). However, while most studies support the view that SARC-CalF has improved the sensitivity of SARC-F, others claim that SARC-CalF has not improved the sensitivity of SARC-F screening (39). Whether it improves the sensitivity of SARC-F or not, ARC-CalF is generally a good choice for the early screening of sarcopenia and can improve the overall diagnostic accuracy of SARC-F.

\section{Gait speed}

There are many methods to detect muscular function in cases of sarcopenia, including the short physical performance battery (SPPB), functional reach test (FRT), Berg balance scale (BBS), stair climb power test (SCPT), and performance activities of daily living (PAD) test (40). SPPB includes the daily pace assessment, timed get-up and go (TGUG), and 6-minute walking test (SMWT) (41). Gait speed, a commonly used index to evaluate muscle function, is considered a fast, safe, and reliable examination method. Evaluating gait speed involves allowing the subject to walk a short distance (3-6 m), selecting the maximum step speed within the distance, and calculating the average step speed in $\mathrm{m} / \mathrm{s}$. Gait speed has been widely used in practice as an important method for the early screening of sarcopenia (42). Some studies strongly recommend gait speed as an independent predictor of mortality (43).

EWGSOP suggests that gait speed should be measured first when conducting an epidemiological investigation of sarcopenia because this is the simplest and most reliable evaluation. In EWGSOP's procedure, the tangent point value of $\leq 0.8 \mathrm{~m} / \mathrm{s}$ over $4 \mathrm{~m}$ is used to measure gait speed (3). Then, the hand grip strength or muscle quality should be evaluated according to the gait speed results. For slow walkers, a muscle quality assessment completes the evaluation of sarcopenia. For fast walkers, it is also suggested that low muscle strength should be defined by the hand grip strength test followed by evaluation of the final muscle quality. FNIH, which is currently the largest sarcopenia project, recommends that in the sequence of screening and case identification, body function should be first checked by gait speed, followed by an evaluation of hand grip strength, and finally muscle quality should be evaluated by DXA (44). The normal gait speed recommended by FNIH is $\leq 0.8 \mathrm{~m} / \mathrm{s}$ 
over $4 \mathrm{~m}$, which is the same as the gait speed recommended by EWGSOP and AWGS (45). At the beginning of 2018, EWGSOP2 updated its original definition to reflect the scientific and clinical evidence accumulated over the past 10 years and suggests the use of a single cut-off speed of $\leq 0.8 \mathrm{~m} / \mathrm{s}$ over $4 \mathrm{~m}$ as the index of severe sarcopenia (27). A slow pace is considered a predictor of disability, cognitive impairment, a need for institutionalization, falls, and mortality, and these factors are significantly associated with sarcopenia $(46,47)$. Because the slowdown of gait speed mostly occurs in the middle-aged and elderly, the current research on gait speed commonly uses a distance of $4 \mathrm{~m}$ or $6 \mathrm{~m}$. A study on obesity and sarcopenic obesity (SO) showed that except for gait speed $(\mathrm{P}<0.001)$, there was no significant difference in sociodemographic, health-related, or functional variables between the SO group and the obesity group (48). Importantly, only gait speed had a significant predictive power for $\mathrm{SO}$ in the elderly $(\beta=-1.906$; $\mathrm{P}<0.0001$; OR 0.149; 95\% CI: 0.051-0.434); therefore, gait speed may be a very useful tool for the early screening of sarcopenia. Because the cut-off point of gait speed proposed by various sarcopenic working groups is based on the results of large data and may not be applicable to specific populations, a cut-off point of gait speed suitable for a given study should be formulated specifically for distinct demographics.

\section{Body mass index (BMI)}

One main evaluation method for sarcopenia muscle quality is the squared ratio of limb skeletal muscle mass $(\mathrm{kg})$ to height (m) as proposed by EWGSOP (3), AWGS (45), and IWGS (49), where if an individual is two or more standard deviations lower than a healthy young individual of the same sex, they are determined to have sarcopenia. Other methods include the ratio of limb lean mass (ALM) to BMI as proposed by FNIH (44), and the ratio of hand grip to BMI, which combined are considered the current "best practices" to define low muscle mass in the elderly. Therefore, BMI plays an important role in sarcopenia diagnosis as a decrease of skeletal muscle mass due to sarcopenia will lead to a decrease of body weight and therefore a decrease of BMI. Lower BMI levels are often accompanied by decreased muscle cell volume, decreased cell number, and atrophy, which decreases muscle mass and muscle strength in patients with muscle attenuation syndrome. Studies have found that a low BMI, low physical activity, and poor nutritional status are all risk factors for sarcopenia, and a multivariate logistic regression found that low $\mathrm{BMI}$ had predictive significance for sarcopenia (50-52). Graf et al. (53) found that the risk of low muscle mass, as measured by BIA, increased when BMI fell to $<18.5 \mathrm{~kg} / \mathrm{m}^{2}$. Landi et al. (54) found that elderly individuals with a $\mathrm{BMI}>21.0 \mathrm{~kg} / \mathrm{m}^{2}$ had a lower risk of sarcopenia than elderly individuals with a BMI $<21.0 \mathrm{~kg} / \mathrm{m}^{2}$ (OR 0.76; 95\% CI: 0.64-0.90). Another study of 285 elderly people found that BMI was associated with sarcopenia when below $22 \mathrm{~kg} / \mathrm{m}^{2}$ (OR 5.973; 95\% CI: 2.932-12.165) (55). Therefore, we consider BMI as a predictor of sarcopenia. If such studies were extended to all regions and ethnic groups, suitable BMI cut-off points for different ethnic groups in different regions could be obtained and used as an initial screening condition in epidemiological investigations.

The measurement method of BMI is simple and easy to implement, which makes it convenient to identify and discover possible sarcopenia populations. This would achieve the goals of early detection, diagnosis, and treatment and improve the quality of life and physical function of elderly patients with sarcopenia. The incidence of sarcopenia and SO shows a rejuvenation trend (56). The ratio of hand grip to $\mathrm{BMI}$ can also be used to predict the presence of SO in children, and because it is cheap and fast, it is especially suitable for community epidemiological investigations that can affect paediatric health interventions. $\mathrm{BMI}$ can also be combined with age to establish a prediction model for screening of sarcopenia. Although it has been proven that such prediction models have good accuracy, further research is still needed to evaluate their specificity and sensitivity (57). BMI can be conveniently used for the preliminary prediction of patients with sarcopenia, which is beneficial to the intervention and control of sarcopenia in the elderly and has a certain reference value for preliminary judgements of whether an individual is suffering from sarcopenia.

\section{Ultrasound (US)}

The use of ultrasound technology has gradually extended from cardiovascular disease to musculoskeletal diseases including sarcopenia (58). A systematic review showed that US was a reliable and effective tool to evaluate the muscle size of the elderly and a feasible method to predict lean body weight (59). Muscle thickness, as measured by US, has a good correlation with muscle volume as measured by DXA, which is the gold standard of measurement for muscle volume (60). A study based on US also found that the feather angle of the medial and lateral head of the gastrocnemius was related to lower limb function in women (61). 
Compared with systemic muscle changes, local muscle changes can improve the diagnosis of sarcopenia in the elderly. Muscle mass, which is defined as muscle strength per unit cross-sectional area of muscle, is determined by the percentage of contractile tissue in the whole muscle. The more contractile tissue there is, the greater the muscle strength per unit area there is that can be sustained, which is closely related to muscle function (61). Rech et al. (62) found that echo intensity measured by US could reveal information about lipid droplet infiltration and fibrosis and that the quality of the muscle could be judged by this information. Muscle thickness as measured by US has been identified as a factor related to low skeletal muscle mass, and the relationship between muscle thickness of various parts of the body and low muscle mass or sarcopenia is becoming a heavily researched subject. If the gastrocnemius muscle thickness is less than $1.5 \mathrm{~cm}$, the muscle quality can be considered low (63), while thigh muscle thickness less than $3.6 \mathrm{~cm}$ for men and $3.4 \mathrm{~cm}$ for women is considered a critical value of muscle loss (64). The critical point of rectus femoris muscle (RFM) thickness is $0.7 \mathrm{~cm}$ for women and $0.9 \mathrm{~cm}$ for men (65). Therefore, the correlation between muscle thickness and sarcopenia in different parts of people of different ages and genders can be further studied in the future, and then, a complete diagnostic cut point can be established. Although there are limitations to the use of US in large-scale epidemiological and community surveys, it can be used as the main screening method for patients with sarcopenia in hospitals, and US screening can also be used to track the prognosis of related diseases associated with sarcopenia.

In addition, the relationship between sarcopenia and handgrip decrease (33), dysphagia (sarcopenic dysphagia) (66), has been determined and is worth using a specific approach in clinical practice.

\section{Early intervention in possible sarcopenia population}

Early intervention methods of individuals at high risk for sarcopenia include health education, exercise intervention, nutrition strategy, and Electro-Myo-Stimulation (EMS).

\section{Health education}

The incidence of sarcopenia is high in the elderly population. It is expected that by 2050 , the prevalence of sarcopenia in the elderly in China will exceed 500 million, and sarcopenia can interact with chronic diseases such as cardiovascular disease, COPD, diabetes, osteoporosis, and other psychological diseases $(4,27)$. Sarcopenia will also be an important health problem for the elderly in the future. After screening out possible sarcopenia population through the above early screening methods, health education should be conducted to inform them of the severe situation of sarcopenia to ensure that they can scientifically understand the serious harm of sarcopenia through both chronic diseases (associated with the elderly) and quality of life and thus take positive countermeasures (67). Presently, there is no standard treatment scheme for sarcopenia at home and abroad, but appropriate coping strategies can be selected according to specific conditions of possible sarcopenia that can prevent and reverse its development.

\section{Exercise intervention}

Exercise is not only a treatment for sarcopenia but also a preventive measure. Moreover, compared with other intervention methods, physical exercise plays the most active role in the management and intervention of sarcopenia and its adverse health consequences (68). A prospective cohort study suggested that the regular occurrence of 30 or more minutes of moderate to intense physical activity per day might be helpful to reduce the risk of severe sarcopenia and SO (69). The LIFE study showed that the probability of serious falls in the 70- to 89-year-old demographic could be significantly reduced using structured moderate-intensity training for approximately 2 years (70). The study also found that the rate of serious dyskinesia in the elderly was significantly reduced after training. Both aerobic exercise and resistance training can reduce the decline in muscle mass and muscle strength with age. Aerobic exercise is also an important strategy to improve the aerobic capacity of muscles in the elderly by initiating mitochondrial adaptation, enhancing cardiovascular function (such as increasing stroke volume), and increasing the capillary density of muscle tissue (71). High-intensity resistance training can effectively promote an increase in muscle strength and the quality of life of the elderly and promote skeletal muscle changes, many of which may help prevent or reverse sarcopenia. High-intensity resistance training is one of the best non-drug intervention methods for the clinical intervention of sarcopenia and has a good synergistic effect with aerobic exercise $(72,73)$. A systematic review showed that exercise intervention could significantly improve strength, balance, and muscle quality, 
but there was a large inter-patient variation in the results due to the heterogeneity of the exercise mode, duration, and intensity (74). Exercise interventions may play a role in improving muscle mass, muscle strength, and walking speed in three months of intervention (75). Therefore, it is necessary to adopt corresponding exercise strategies for different people with sarcopenia to improve the results of exercise intervention. At present, individualized exercise prescription therapy has become widely utilized, but there is currently no exercise prescription for possible sarcopenia. If exercise prescription therapy can be applied to the clinical intervention of sarcopenia, then it will guide possible sarcopenia populations. Purposeful, planned, and scientific exercise can be crucial in the prevention and treatment of sarcopenia.

Recently, studies confirmed that sarcopenia caused by ageing does not progress at the same speed in all regions of the body, e.g., the decrease in the speed of the muscles of the lower limbs is faster than that of the upper limbs (76). Sometimes the muscle mass of the upper limbs of the elderly will even increase due to compensation (77). Therefore, the analysis of muscle changes in different parts of the body not only helps in diagnosing possible sarcopenia but also in devising a targeted plan for precisely exercising local muscles and improving local muscle function.

\section{Nutrition strategy}

The possible sarcopenia population should give priority to high-protein (whey protein or animal protein), nutrientrich, and energy-rich semi-fluid and fluid foods and supplement vitamin $\mathrm{D}$ and omega-3 polyunsaturated fatty acids (n-3 PUFAs) at appropriate amounts. This population should also increase or reduce their intake as needed to maintain their nutrition and water electrolyte balance and appropriately supplement minerals, such as magnesium, selenium, and calcium. This approach seems to help resist or prevent sarcopenia (78-80). In some studies, dietary interventions involving protein supplementation improved muscle function and/or strength, while other dietary methods were less effective (81). Therefore, the preventive effects of a diet rich in protein and conforming to conventional dietary habits on sarcopenia deserve more attention. Adequate protein intake can also increase muscle quality and some muscle functions. Currently, the minimum protein intake required to maintain muscle quality in the elderly is estimated to at $0.8 \mathrm{~g} / \mathrm{kg} /$ day, and muscle quality is improved when the daily protein intake is $1.0-1.2 \mathrm{~g} / \mathrm{kg}(68,82)$. Among nutrients in the human diet, amino acids can directly trigger the synthesis of muscle proteins, especially leucine. Leucine-rich essential amino acids help overcome the anabolic resistance of the elderly and effectively stimulates the synthesis of muscle proteins (83). A clinical meta-study on the therapeutic significance of diet and exercise for sarcopenia showed that the synthesis of skeletal muscle protein in the elderly increased and muscle function improved after eating leucine-rich food (84). Moreover, the leucine metabolite $\beta$-hydroxy- $\beta$-methyl butyrate (HMB) has also proven effective in treating muscle atrophy, increasing muscle cell production, reducing muscle cell apoptosis, and shows a positive effect on muscle protein turnover (85). For possible sarcopenia population, nutritional supplementation can not only delay or prevent the development of sarcopenia but also further improve the health status of individuals.

Presently, vitamin D is also considered a basic treatment for sarcopenia $(86,87)$. Hirani et al. found that serum concentrations of vitamins $1,25 \mathrm{D}$ and $25 \mathrm{D}$ lower than baseline levels were independently related to the incidence of sarcopenia in the following 5 years, and maintaining adequate levels of vitamin $\mathrm{D}$ reduced the incidence of muscular dystrophy in elderly men (88). The latest evidence shows that supplementation of 800-1,000 international units of vitamin D per day, especially when vitamin $\mathrm{D}$ content is low, and taking approximately $3 \mathrm{~g}$ of $\mathrm{n}-3$ PUFAs per day may be beneficial to body function, muscle quality, and strength $(89,90)$. In addition, the combined supplementation of protein, leucine, vitamin $D$, and $n-3$ PUFAs may bring more benefits and may represent a nutritional intervention strategy to prevent sarcopenia. Although exercise can stimulate decomposition in muscle proteins, under a reasonable diet, the increase in the net balance of protein decomposition and synthesis after exercise will lead to an increase of muscle protein and muscle hypertrophy and can also continuously improve the strength of lower limb muscles $(81,91)$. Therefore, nutrition strategy and exercise strategy are the main synthetic stimuli that lead to muscle protein synthesis (92). A meta-analysis showed that the combination of exercise and nutrition intervention within three months may have a positive effect on improving walking speed (75). A promising strategy to prevent or delay the onset of sarcopenia may be to optimize the potential of muscle protein synthesis by taking an appropriate combination of supplements and engaging in physical exercise. Future studies with larger sample sizes should verify this theory. 


\section{EMS}

Although exercise is the cornerstone of intervention for sarcopenia, nutrition strategy alone is not enough for people with disabilities or diseases that prevent them from engaging in any exercise or reaching the recommended exercise dose to affect strength or muscle quality. Therefore, EMS may be a feasible choice to increase muscle quality, save time, and preserve physical strength with minimal damage to the joints (93-95). The most common and scientifically verified form of EMS relies on bipolar, low frequency $(50-100 \mathrm{~Hz})$ myoelectric stimulation with medium pulse widths (300-400 $\mu$ s). For 20 minutes per week, up to 10 regions or 14 muscle groups can be activated and up to $2,800 \mathrm{~cm}^{2}$ of different regions can be simultaneously activated; in addition, the intensity of each region can be independently selected (96). EMS has been recognized as an effective alternative exercise technology, which can significantly affect muscle and fat mass, improve sarcopenia and $\mathrm{SO}$ in the elderly over 70 years old, and shows a high compliance rate (97). Most EMS research shows that the lean body weight and fat mass of the elderly have a significant increase after EMS. Moreover, due to its high time efficiency and user-friendliness, EMS may be the best intervention choice to replace exercise strategies, and it is an effective and safe method to prevent cardiovascular metabolic risk factors and diseases in elderly women who are unable or unwilling to exercise regularly (98). For some people, the application of EMS is an alternative to exercise intervention.

A systematic review of randomized clinical trials showed that EMS could be an effective and safe solution for sarcopenia in terms of muscle mass, size, and performance, fat distribution, and strength parameters both locally and globally. EMS should be considered for use in elderly patients with sarcopenia in addition to other possible methods (99). However, there is still controversy over the effect of the combination of nutritional intervention and EMS. Some studies found a synergistic effect when EMS was used in combination with moderate-to-high doses of whey protein supplements $(100,101)$, while others found that protein-rich supplements did not increase the effect of EMS (98). With the rapid development of technology, EMS has very wide prospects for application and can be used as a combined intervention method in sports and nutrition intervention.

\section{Conclusions}

In summary, sarcopenia is likely to attract more attention as problems of the population ageing intensify. Early screening and intervention for sarcopenia can greatly reduce expenditures on national public health. The accuracy of early screening for sarcopenia should be tested, and more research should focus on exploring simple, accurate, and fast methods to achieve early screening for sarcopenia. It is especially important to apply effective combined intervention measures to possible sarcopenia to reduce its adverse consequences and the extent of harm.

\section{Acknowledgments}

Funding: This review was supported by National Key R\&D Program of China (2019YFA0111900), National Natural Science Foundation of China (81874030, 81871096), Provincial Natural Science Foundation of Hunan (2020JJ3060, 2019JJ50949, 2018JJ2636), InnovationDriven Project of Central South university (2020CX045), Wu Jieping Medical Foundation (320.6750.2020-03-14), CMA.Young and Middle-aged Doctors Outstanding Development Program-Osteoporosis Specialized Scientific Research Fund Project (G-X-2019-1107-12), Undergraduate Innovation Training Program of Central South University (No. XCX20190545, XCX20190606), the Key Research and Development Program of Hunan Province (2018SK2076) and the program of Health Commission of Hunan Province (20201359, 20201902, B2019187).

\section{Footnote}

Reporting Checklist: The authors have completed the NARRATIVE REVIEW reporting checklist. Available at http://dx.doi.org/10.21037/apm-20-967

Conflicts of Interest: All authors have completed the ICMJE uniform disclosure form (available at http://dx.doi. org/10.21037/apm-20-967). The authors have no conflicts of interest to declare.

Ethical Statement: The authors are accountable for all aspects of the work in ensuring that questions related to the accuracy or integrity of any part of the work are appropriately investigated and resolved.

Open Access Statement: This is an Open Access article distributed in accordance with the Creative Commons Attribution-NonCommercial-NoDerivs 4.0 International 
License (CC BY-NC-ND 4.0), which permits the noncommercial replication and distribution of the article with the strict proviso that no changes or edits are made and the original work is properly cited (including links to both the formal publication through the relevant DOI and the license). See: https://creativecommons.org/licenses/by-nc-nd/4.0/.

\section{References}

1. Rosenberg IH. Sarcopenia: origins and clinical relevance. J Nutr 1997;127:990S-1S.

2. Baumgartner RN, Koehler KM, Gallagher D, et al. Epidemiology of sarcopenia among the elderly in New Mexico. Am J Epidemiol 1998;147:755-63.

3. Cruz-Jentoft AJ, Baeyens JP, Bauer JM, et al. Sarcopenia: European consensus on definition and diagnosis: Report of the European Working Group on Sarcopenia in Older People. Age Ageing 2010;39:412-23.

4. Cruz-Jentoft AJ, Sayer AA. Sarcopenia. Lancet 2019;393:2636-46.

5. Anker SD, Morley JE, von Haehling S. Welcome to the ICD-10 code for sarcopenia. J Cachexia Sarcopenia Muscle 2016;7:512-4.

6. Sakamoto K. Progress of sarcopenia research. Nihon Yakurigaku Zasshi 2017;149:186.

7. Wen X, An P, Chen WC, et al. Comparisons of sarcopenia prevalence based on different diagnostic criteria in Chinese older adults. J Nutr Health Aging 2015;19:342-7.

8. Yoo JI, Ha YC, Kwon HB, et al. High Prevalence of Sarcopenia in Korean Patients after Hip Fracture: a CaseControl Study. J Korean Med Sci 2016;31:1479-84.

9. Mori H, Tokuda Y. Differences and overlap between sarcopenia and physical frailty in older communitydwelling Japanese. Asia Pac J Clin Nutr 2019;28:157-65.

10. Ethgen O, Beaudart C, Buckinx F, et al. The Future Prevalence of Sarcopenia in Europe: A Claim for Public Health Action. Calcif Tissue Int 2017;100:229-34.

11. Morley JE, Anker SD, von Haehling S. Prevalence, incidence, and clinical impact of sarcopenia: facts, numbers, and epidemiology-update 2014. J Cachexia Sarcopenia Muscle 2014;5:253-9.

12. Palus S, Springer JI, Doehner W, et al. Models of sarcopenia: Short review. Int J Cardiol 2017;238:19-21.

13. Woo J. Sarcopenia. Clin Geriatr Med 2017;33:305-14.

14. Marty E, Liu Y, Samuel A, et al. A review of sarcopenia: Enhancing awareness of an increasingly prevalent disease. Bone 2017;105:276-86.

15. Mohd Nawi SN, Khow KS, Lim WS, et al. Screening
Tools for Sarcopenia in Community-Dwellers: A Scoping Review. Ann Acad Med Singapore 2019;48:201-16.

16. Miller J, Wells L, Nwulu U. Validated screening tools for the assessment of cachexia, sarcopenia, and malnutrition: a systematic review. Am J Clin Nutr 2018;108:1196-208.

17. Jeon JY, Wang HJ, Ock SY, et al. Newly Developed Sarcopenia as a Prognostic Factor for Survival in Patients who Underwent Liver Transplantation. PLoS One 2015;10:e143966.

18. Onoue Y, Izumiya Y, Hanatani S, et al. A simple sarcopenia screening test predicts future adverse events in patients with heart failure. Int J Cardiol 2016;215:301-6.

19. Malmstrom TK, Morley JE. SARC-F: a simple questionnaire to rapidly diagnose sarcopenia. J Am Med Dir Assoc 2013;14:531-2.

20. Bahat G, Yilmaz O, Kilic C, et al. Performance of SARC-F in Regard to Sarcopenia Definitions, Muscle Mass and Functional Measures. J Nutr Health Aging 2018;22:898-903.

21. Kemmler W, Sieber C, Freiberger E, et al. The SARC-F Questionnaire: Diagnostic Overlap with Established Sarcopenia Definitions in Older German Men with Sarcopenia. Gerontology 2017;63:411-6.

22. Ida S, Kaneko R, Murata K. SARC-F for Screening of Sarcopenia Among Older Adults: A Meta-analysis of Screening Test Accuracy. J Am Med Dir Assoc 2018;19:685-9.

23. Woo J, Leung J, Morley JE. Validating the SARC-F: a suitable community screening tool for sarcopenia?J Am Med Dir Assoc 2014;15:630-4.

24. Li M, Kong Y, Chen H, et al. Accuracy and prognostic ability of the SARC-F questionnaire and Ishii's score in the screening of sarcopenia in geriatric inpatients. Braz J Med Biol Res 2019;52:e8204.

25. Yang M, Hu X, Xie L, et al. Comparing Mini Sarcopenia Risk Assessment With SARC-F for Screening Sarcopenia in Community-Dwelling Older Adults. J Am Med Dir Assoc 2019;20:53-7.

26. Dent E, Morley JE, Cruz-Jentoft AJ, et al. International Clinical Practice Guidelines for Sarcopenia (ICFSR): Screening, Diagnosis and Management. J Nutr Health Aging 2018;22:1148-61.

27. Cruz-Jentoft AJ, Bahat G, Bauer J, et al. Sarcopenia: revised European consensus on definition and diagnosis. Age Ageing 2019;48:16-31.

28. Rolland Y, Dupuy C, Abellan Van Kan G, et al. Sarcopenia Screened by the SARC-F Questionnaire and Physical Performances of Elderly Women: A Cross-Sectional 
Study. J Am Med Dir Assoc 2017;18:848-52.

29. Kotlarczyk MP, Perera S, Nace DA, et al. Identifying Sarcopenia in Female Long-Term Care Residents: A Comparison of Current Guidelines. J Am Geriatr Soc 2018;66:316-20.

30. Gómez-Tabares G, Garcia W, Bedoya-Dorado E, et al. Screening sarcopenia through SARC-F in postmenopausal women: a single-center study from South America. Climacteric 2019;22:627-31.

31. Woo J, Yu R, Leung J. A 3-Item SARC-F. J Am Med Dir Assoc 2018;19:223-8.

32. Yang $M, H u X$, Xie L, et al. SARC-F for sarcopenia screening in community-dwelling older adults: Are 3 items enough? Medicine (Baltimore) 2018;97:e11726.

33. Barbosa-Silva TG, Menezes AM, Bielemann RM, et al. Enhancing SARC-F: Improving Sarcopenia Screening in the Clinical Practice. J Am Med Dir Assoc 2016;17:1136-41.

34. Kim S, Kim M, Lee Y, et al. Calf Circumference as a Simple Screening Marker for Diagnosing Sarcopenia in Older Korean Adults: the Korean Frailty and Aging Cohort Study (KFACS). J Korean Med Sci 2018;33:e151.

35. Chen LK, Woo J, Assantachai P, et al. Asian Working Group for Sarcopenia: 2019 Consensus Update on Sarcopenia Diagnosis and Treatment. J Am Med Dir Assoc 2020;21:300-7.e2.

36. Yang $\mathrm{M}, \mathrm{Lu} \mathrm{J}$, Jiang J, et al. Comparison of four sarcopenia screening tools in nursing home residents. Aging Clin Exp Res 2019;31:1481-9.

37. Yang $M, H u X$, Xie L, et al. Screening Sarcopenia in Community-Dwelling Older Adults: SARC-F vs SARC-F Combined With Calf Circumference (SARC-CalF). J Am Med Dir Assoc 2018;19:277.e1-8.

38. Mo Y, Dong X, Wang XH. Screening Accuracy of SARC-F Combined With Calf Circumference for Sarcopenia in Older Adults: A Diagnostic Meta-Analysis. J Am Med Dir Assoc 2020;21:288-9.

39. Bahat G, Oren MM, Yilmaz O, et al. Comparing SARC-F with SARC-CalF to Screen Sarcopenia in Community Living Older Adults. J Nutr Health Aging 2018;22:1034-8.

40. Thomas DR. Arquivos Brasileiros de Endocrinologia \& Metabologia. Arq Bras Endocrinol Metab 2014;58:464-9.

41. Cawthon PM. Assessment of Lean Mass and Physical Performance in Sarcopenia. J Clin Densitom 2015;18:467-71.

42. Bruyere O, Beaudart C, Reginster JV, et al. Assessment of muscle mass, muscle strength and physical performance in clinical practice: an international survey. Eur Geriatr Med
2016;7:243-6.

43. Abe T, Kitamura A, Taniguchi Y, et al. Pathway from gait speed to incidence of disability and mortality in older adults: A mediating role of physical activity. Maturitas 2019;123:32-6.

44. Studenski SA, Peters KW, Alley DE, et al. The FNIH sarcopenia project: rationale, study description, conference recommendations, and final estimates. J Gerontol A Biol Sci Med Sci 2014;69:547-58.

45. Chen LK, Liu LK, Woo J, et al. Sarcopenia in Asia: consensus report of the Asian Working Group for Sarcopenia. J Am Med Dir Assoc 2014;15:95-101.

46. Sanders JB, Bremmer MA, Comijs HC, et al. Gait Speed and Processing Speed as Clinical Markers for Geriatric Health Outcomes. Am J Geriatr Psychiatry 2017;25:374-85.

47. Peel NM, Kuys SS, Klein K. Gait speed as a measure in geriatric assessment in clinical settings: a systematic review. J Gerontol A Biol Sci Med Sci 2013;68:39-46.

48. CM Santos, JMD Dias, GAR Samora, et al. Prevalence of obesity, sarcopenic obesity and associated factors: A FIBRA Network study. Fisioterapia em Movimento 2017;30:S161-9.

49. Chumlea WC, Cesari M, Evans WJ, et al. International working group on Sarcopenia. J Nutr Health Aging 2011;15:450-5.

50. Yalcin A, Aras S, Atmis V, et al. Sarcopenia prevalence and factors associated with sarcopenia in older people living in a nursing home in Ankara Turkey. Geriatr Gerontol Int 2016;16:903-10.

51. Senior HE, Henwood TR, Beller EM, et al. Prevalence and risk factors of sarcopenia among adults living in nursing homes. Maturitas 2015;82:418-23.

52. Han P, Zhao J, Guo Q, et al. Incidence, Risk Factors, and the Protective Effect of High Body Mass Index against Sarcopenia in Suburb-Dwelling Elderly Chinese Populations. J Nutr Health Aging 2016;20:1056-60.

53. Graf CE, Pichard C, Herrmann FR, et al. Prevalence of low muscle mass according to body mass index in older adults. Nutrition 2017;34:124-9.

54. Landi F, Liperoti R, Fusco D, et al. Prevalence and risk factors of sarcopenia among nursing home older residents. J Gerontol A Biol Sci Med Sci 2012;67:48-55.

55. Bravo-José P, Moreno E, Espert M, et al. Prevalence of sarcopenia and associated factors in institutionalised older adult patients. Clin Nutr ESPEN 2018;27:113-9.

56. Steffl M, Chrudimsky J, Tufano JJ. Using relative handgrip strength to identify children at risk of sarcopenic obesity. PLoS One 2017;12:e0177006. 
57. Goodman MJ, Ghate SR, Mavros P, et al. Development of a practical screening tool to predict low muscle mass using NHANES 1999-2004. J Cachexia Sarcopenia Muscle 2013;4:187-97.

58. Stringer HJ, Wilson D. The Role of Ultrasound as a Diagnostic Tool for Sarcopenia. J Frailty Aging 2018;7:258-61.

59. Nijholt $W$, Scafoglieri A, Jager-Wittenaar H, et al. The reliability and validity of ultrasound to quantify muscles in older adults: a systematic review. J Cachexia Sarcopenia Muscle 2017;8:702-12.

60. Zhu S, Lin W, Chen S, et al. The correlation of muscle thickness and pennation angle assessed by ultrasound with sarcopenia in elderly Chinese community dwellers. Clin Interv Aging 2019;14:987-96.

61. Ticinesi A, Narici MV, Lauretani F, et al. Assessing sarcopenia with vastus lateralis muscle ultrasound: an operative protocol. Aging Clin Exp Res 2018;30:1437-43.

62. Rech A, Radaelli R, Goltz FR, et al. Echo intensity is negatively associated with functional capacity in older women. Age (Dordr) 2014;36:9708.

63. Wang J, Hu Y, Tian G. Ultrasound measurements of gastrocnemius muscle thickness in older people with sarcopenia. Clin Interv Aging 2018;13:2193-9.

64. Hida T, Ando K, Kobayashi K, et al. Ultrasound measurement of thigh muscle thickness for assessment of sarcopenia. Nagoya J Med Sci 2018;80:519-27.

65. Rustani K, Kundisova L, Capecchi PL, et al. Ultrasound measurement of rectus femoris muscle thickness as a quick screening test for sarcopenia assessment. Arch Gerontol Geriatr 2019;83:151-4.

66. Fujishima I, Fujiu-Kurachi M, Arai H, et al. Sarcopenia and dysphagia: Position paper by four professional organizations. Geriatr Gerontol Int 2019;19:91-7.

67. Tsekoura M, Kastrinis A, Katsoulaki M, et al. Sarcopenia and Its Impact on Quality of Life. Adv Exp Med Biol 2017;987:213-8.

68. Liguori I, Russo G, Aran L, et al. Sarcopenia: assessment of disease burden and strategies to improve outcomes. Clin Interv Aging 2018;13:913-27.

69. Aggio DA, Sartini C, Papacosta O, et al. P135? Association of objectively measured physical activity and sedentary time with sarcopenia, severe sarcopenia and sarcopenic obesity in older men. J Epidemiol Community Health 2016;70:A113.

70. Bann D, Chen H, Bonell C, et al. Socioeconomic differences in the benefits of structured physical activity compared with health education on the prevention of major mobility disability in older adults: the LIFE study. J Epidemiol Community Health 2016;70:930-3.

71. Trouwborst I, Verreijen A, Memelink R, et al. Exercise and Nutrition Strategies to Counteract Sarcopenic Obesity. Nutrients 2018;10:E605.

72. Chen HT, Chung YC, Chen YJ, et al. Effects of Different Types of Exercise on Body Composition, Muscle Strength, and IGF-1 in the Elderly with Sarcopenic Obesity. J Am Geriatr Soc 2017;65:827-32.

73. Zembroń-Łacny A, Dziubek W, Rogowski L, et al. Sarcopenia: monitoring, molecular mechanisms, and physical intervention. Physiol Res 2014;63:683-91.

74. Vlietstra L, Hendrickx W, Waters DL. Exercise interventions in healthy older adults with sarcopenia: A systematic review and meta-analysis. Australas J Ageing 2018;37:169-83.

75. Yoshimura Y, Wakabayashi H, Yamada M, et al. Interventions for Treating Sarcopenia: A Systematic Review and Meta-Analysis of Randomized Controlled Studies. J Am Med Dir Assoc 2017;18:553.e1-16.

76. Fukumoto Y, Ikezoe T, Yamada Y, et al. Age-Related Ultrasound Changes in Muscle Quantity and Quality in Women. Ultrasound Med Biol 2015;41:3013-7.

77. Ata AM, Kara M, Kaymak B, et al. Regional and total muscle mass, muscle strength and physical performance: The potential use of ultrasound imaging for sarcopenia. Arch Gerontol Geriatr 2019;83:55-60.

78. van Dronkelaar C, Van Velzen A, Abdelrazek M, et al. Minerals and Sarcopenia; The Role of Calcium, Iron, Magnesium, Phosphorus, Potassium, Selenium, Sodium, and Zinc on Muscle Mass, Muscle Strength, and Physical Performance in Older Adults: A Systematic Review. J Am Med Dir Assoc 2018;19:6-11.e3.

79. Cruz-Jentoft AJ, Kiesswetter E, Drey M, et al. Nutrition, frailty, and sarcopenia. Aging Clin Exp Res 2017;29:43-8.

80. Dupont J, Dedeyne L, Dalle S, et al. The role of omega-3 in the prevention and treatment of sarcopenia. Aging Clin Exp Res 2019;31:825-6.

81. Anton SD, Hida A, Mankowski R, et al. Nutrition and Exercise in Sarcopenia. Curr Protein Pept Sci 2018;19:649-67.

82. Gaffney-Stomberg E, Insogna KL, Rodriguez NR, et al. Increasing dietary protein requirements in elderly people for optimal muscle and bone health. J Am Geriatr Soc 2009;57:1073-9.

83. Kobayashi H. Amino Acid Nutrition in the Prevention and Treatment of Sarcopenia. Yakugaku Zasshi 2018;138:1277-83. 
84. Cruz-Jentoft AJ, Landi F, Schneider SM, et al. Prevalence of and interventions for sarcopenia in ageing adults: a systematic review. Report of the International Sarcopenia Initiative (EWGSOP and IWGS). Age Ageing 2014;43:748-59.

85. Rossi AP, D'Introno A, Rubele $S$, et al. The Potential of $\beta$-Hydroxy- $\beta$-Methylbutyrate as a New Strategy for the Management of Sarcopenia and Sarcopenic Obesity. Drugs Aging 2017;34:833-40.

86. Morley JE. Frailty and Sarcopenia: The New Geriatric Giants. Rev Invest Clin 2016;68:59-67.

87. Morley JE. Pharmacologic Options for the Treatment of Sarcopenia. Calcif Tissue Int 2016;98:319-33.

88. Hirani V, Cumming RG, Naganathan V, et al. Longitudinal Associations Between Vitamin D Metabolites and Sarcopenia in Older Australian men: The Concord Health and Aging in Men Project. J Gerontol A Biol Sci Med Sci 2017;73:131-8.

89. Tessier AJ, Chevalier S. An Update on Protein, Leucine, Omega-3 Fatty Acids, and Vitamin D in the Prevention and Treatment of Sarcopenia and Functional Decline. Nutrients 2018;10:E1099.

90. Schneider DA, Trence DL. Possible role of nutrition in prevention of sarcopenia and falls. Endocr Pract 2019;25:1184-90.

91. Xie WQ, Xiao GL, Fan YB, et al. Sarcopenic obesity: research advances in pathogenesis and diagnostic criteria. Aging Clin Exp Res 2019. doi: 10 1007/s40520-01901435-9.

92. Bosaeus I, Rothenberg E. Nutrition and physical activity for the prevention and treatment of age-related sarcopenia. Proc Nutr Soc 2016;75:174-80.

93. Kemmler W, Bebenek M, Engelke K, et al. Impact of whole-body electromyostimulation on body composition in elderly women at risk for sarcopenia: the Training and ElectroStimulation Trial (TEST-III). Age 2014;36:395-406.
94. Kemmler W, von Stengel S. Whole-body electromyostimulation as a means to impact muscle mass and abdominal body fat in lean, sedentary, older female adults: subanalysis of the TEST-III trial. Clin Interv Aging 2013;8:1353-64.

95. Kemmler W, Von Stengel S. Alternative Exercise Technologies to Fight against Sarcopenia at Old Age: A Series of Studies and Review. J Aging Res 2012;2012:109013.

96. Kemmler W, Teschler M, Weissenfels A, et al. WholeBody EMS to Fight Sarcopenic Obesity--a Review with Emphasis on Body Fat. Dtsch Z Sportmed 2017;68:170-7.

97. Kemmler W, Teschler M, Weissenfels A, et al. Wholebody electromyostimulation to fight sarcopenic obesity in community-dwelling older women at risk. Results of the randomized controlled FORMOsA-sarcopenic obesity study. Osteoporos Int 2016;27:3261-70.

98. Wittmann K, Sieber C, von Stengel S, et al. Impact of whole body electromyostimulation on cardiometabolic risk factors in older women with sarcopenic obesity: the randomized controlled FORMOsA-sarcopenic obesity study. Clin Interv Aging 2016;11:1697-706.

99. Chisari E, Pavone V, Sessa G, et al. Electromyostimulation and whole-body vibration effects in elder sarcopenic patients. Muscles Ligaments Tendons J 2019;9:433-41.

100. Kemmler W, Kohl M, Freiberger E, et al. Effect of whole-body electromyostimulation and/or protein supplementation on obesity and cardiometabolic risk in older men with sarcopenic obesity: the randomized controlled FranSO trial. BMC Geriatrics 2018;18:70.

101. Kemmler W, Grimm A, Bebenek M, et al. Effects of Combined Whole-Body Electromyostimulation and Protein Supplementation on Local and Overall Muscle/Fat Distribution in Older Men with Sarcopenic Obesity: The Randomized Controlled Franconia Sarcopenic Obesity (FranSO) Study. Calcif Tissue Int 2018;103:266-77.
Cite this article as: Xie WQ, Xiao GL, Hu PW, He YQ, Lv S, Xiao WF. Possible sarcopenia: early screening and interventionnarrative review. Ann Palliat Med 2020;9(6):4283-4293. doi: 10.21037/apm-20-967 